Postprint: Van De Walle W, Janssen H. 2016. Thermal conductivity prediction model for porous building blocks, Bauphysik, 38(6):340-347.

doi: 10.1002/bapi.201610037

\title{
A THERMAL CONDUCTIVITY PREDICTION MODEL FOR POROUS BUILDING BLOCKS
}

\author{
W. Van De Walle and H. Janssen \\ KU Leuven, Department of Civil Engineering, Building Physics Section \\ Kasteelpark Arenberg 40 - bus 02447, BE-3001 Heverlee (Leuven), Belgium \\ Wouter.VanDeWalle@kuleuven.be
}

\begin{abstract}
A 3D model for the prediction of the effective thermal conductivity of porous building blocks is introduced. Simulations are performed directly on the microstructure using voxel images and the finite element technique. Very good agreement with analytical solutions is achieved. The model is used to investigate the miscalculation effect of 2D simulations, clearly indicating the need for a 3D model. Furthermore, a method for incorporating radiative heat transfer at the microscale is implemented and applied on a synthetic sample, demonstrating the influence of thermal radiation on the effective thermal conductivity.
\end{abstract}

\section{INTRODUCTION}

Highly porous building blocks find frequent use in the construction of buildings because of their relatively high resistance to heat transfer. Typical examples include cellular concrete, cellular glass, and more recently also building blocks based on slag waste and other secondary materials. However, due to increasingly stringent energy regulations, there is an ever growing demand for even better insulating building blocks.

The heat flow through such building materials is usually described at the macroscale with Fourier's law using the effective thermal conductivity (ETC), while in fact it originates from the aggregation of conductive, radiative and convective heat transfer at the microscale. The relative contribution of each of these heat transfer mechanisms depends strongly on the microstructural parameters, i.e. porosity, pore size, matrix connectivity etc. (Carson et al. 2003). A correct understanding of the direct relation between these microstructural parameters and the total heat transport is therefore crucial in the development of improved building blocks. However, current models attempting to study their influence still exhibit large errors due to 2D simplifications, neglect of thermal radiation or their very limited applicability for a restricted class of materials (Randrianalisoa \& Baillis 2014).

This paper presents a newly developed 3D FEM model for simulating the heat transport through a porous structure at the microscale. The first part explains the workflow of the model, followed by a verification study on an elementary pore structure. Subsequently, a method for incorporating radiative heat transfer at the microscale is studied and extended. Finally, in the remainder of the paper, the model is used to make a first study on the discrepancy between $2 \mathrm{D}$ and $3 \mathrm{D}$ simulations of the same sample, and to study the share of thermal radiation on the ETC.

\section{SIMULATION MODEL}

The effective thermal conductivity of a porous material is obtained by simulating the heat transfer through a representative cubic sample at the microscale. The model is subdivided in three steps: (1) obtaining the geometrical representation of the microstructure, (2) creating a finite element mesh and (3) simulating the heat flow through the microstructure. All three steps are explained below. Each step is completely controlled via a set of Matlab routines, hence leading to an automated and easily parameterized workflow. A summarizing overview of the model workflow is shown in Figure 1.

\section{Geometry: 3D voxel image of the microstructure}

The model is based on a $3 \mathrm{D}$ voxel image - the 3D equivalent of a $2 \mathrm{D}$ pixel image - representation of the microstructure. The numeric value of each voxel indicates which material phase is located at that specific location. These voxel images can generally be acquired in two ways: via micro-CT scanning or via synthetic generation techniques.

Using the micro-CT technique, the microstructure of a physical sample is obtained through $\mathrm{x}$-ray imaging and computed tomography. Objects containing features down to several micrometers can be resolved, hence allowing to incorporate the true microstructural properties of the sample. A CT scan voxel image of a cellular concrete is shown in Figure 1a.

Using synthetic generation methods, the $3 \mathrm{D}$ voxel image is constructed in a deterministic or stochastic manner. The technique hence allows for fast designing and testing of new microstructures and a more thorough study of specific microscale parameters. A classic method is the generation and insertion of sphereous pores in a solid material like described by 
Postprint: Van De Walle W, Janssen H. 2016. Thermal conductivity prediction model for porous building blocks,

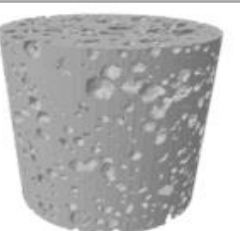

(a) Micro-CT image

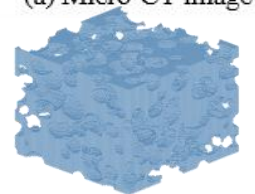

(b) Synthetic generated image

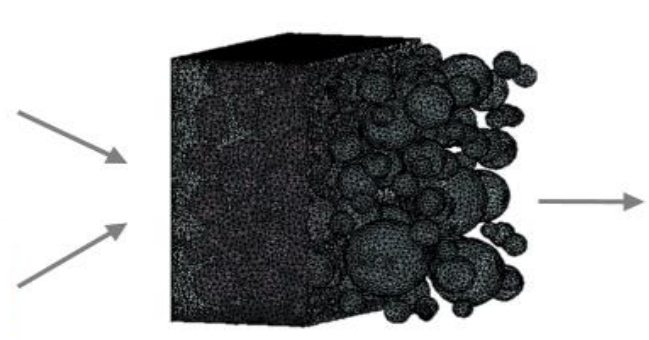

(c) Finite element mesh

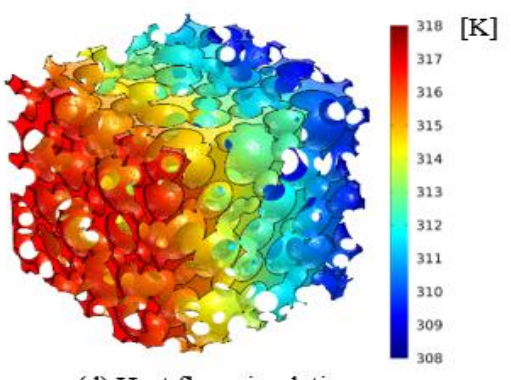

(d) Heat flow simulation

Figure 1: Different steps in the workflow of the model

She et al. (2014). Besides, the discrete voxel image format also allows for the generation of nonanalytically describable pore structures, leading often to much more realistic microstructures. Recent examples can be found in Gaiselmann et al. (2014). For this paper, we have currently implemented a simple sphere generation algorithm based on the method of W. She. An example of a generated structure is shown in Figure $1 \mathrm{~b}$.

Both methods provide a 3D voxel image, with a level of detail depending on the used resolution (the total amount of voxels). A higher resolution should hence be used for resolving smaller features, taking into account the increasing memory usage. Finally, the image can be imported into Matlab as a 3D binary matrix. This allows for an easy manipulation of the sample and the calculation of several microstructural properties like porosity, pore size distribution etc.

\section{Meshing procedure}

A finite element mesh is created from the $3 \mathrm{D}$ voxel image using the open source iso2mesh Matlab toolbox (Fang \& Boas 2009). This provides an extensive set of routines for manipulating the sample and extracting a tetrahedral mesh based on the c++ CGAL library (Alliez et al. 2015). To cope with the complex pore scale geometry, the code makes use of the Delaunay triangulation technique, resulting in a fast generation of relatively qualitative elements. Furthermore, the technique inherently smooths jagged surfaces between the different material phases. We extended the meshing procedure with a point insertion algorithm, leading to a better approximation of the straigth edges of the cubic sample. The density of the mesh is controlled through the set maximum radii for the surface and tetrahedra circumscribing Delaunay ball. A quadratic shape function is used in all the elements. An example mesh is shown in Figure 1c.

\section{Simulation}

The finite element mesh is imported into the COMSOL simulation package to perform heat transfer simulations. The thermal conductivity of the gaseous and solid phase are applied to the respective regions. A method for including thermal radiation is elaborated further in the paper. Natural convection on the other hand can effectively be neglected for pores with a diameter smaller than $4 \mathrm{~mm}$ (Clyne et al. 2006) and is hence not implemented.

A temperature difference of $10 \mathrm{~K}$ is applied between opposing boundaries while the other boundaries are set to adiabatic boundary conditions. The succesive over relaxation (SOR) solver with relative tolerance of $10^{-4}$ is applied, showing good performance for solving the system of equations. An example temperature profile of a sample is shown in Figure 1d.

After the stationary simulation, the ETC can finally be calculated by rewriting the Fourier heat law according to J. Chen et al. (2015) for cubic samples:

$$
\lambda_{\text {eff }}=\frac{\int_{V_{\text {cube }}} q_{x} * d V}{\left(T_{\text {hot }}-T_{\text {cold }}\right) *\left(L_{\text {sample }}\right)^{2}}
$$

$\lambda_{\text {eff }}=$ the effective thermal conductivity of the sample $[\mathrm{W} / \mathrm{mK}] ; V_{\text {cube }}=$ the volume of the cubic sample $\left[\mathrm{m}^{3}\right]$; $q_{x}=$ the heat flux in every element in the direction of the applied temperature gradient $\left[\mathrm{W} / \mathrm{m}^{2}\right] ; T_{\text {hot }} / T_{\text {cold }}=$ the temperature applied at the hot, respectively cold side $[\mathrm{K}] ; L_{\text {sample }}=$ the thickness of the cubic sample.

\section{VERIFICATION OF THE MODEL}

The model is verified using a synthetic sample having identical spherical pores arranged in a lattice of the face-centred cubic (fcc) type. This elementary packing of spheres consists of a repetitive cube having a pore centered on every corner and on every face, as shown in Figure 2b.

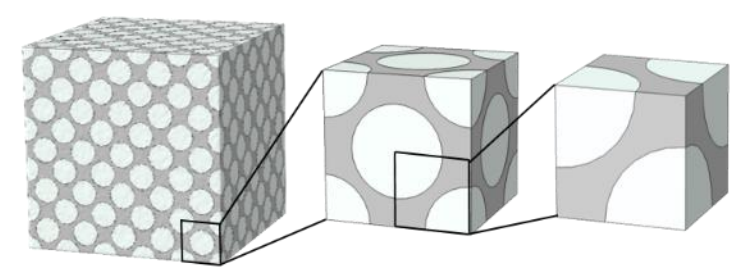

Figure 2: A sample with fcc packing at $50 \%$ porosity $(a)$, the repetitive cube (b), and the unit cell (c). 
Postprint: Van De Walle W, Janssen H. 2016. Thermal conductivity prediction model for porous building blocks,

Although the heat flow through such a repetitive cube is - due to the perfect symmetry - representative for larger arrangements, a larger sample is generated to demonstrate the possibilities of the model for working with larger datasets. A cubic sample consisting of $5 \times 5 \times 5$ of these representative cubes is generated, following the recommendations of Chen et al. (2015) on the size of a representative volume for heat transfer in random microstructures. Samples at 5 different porosities between $10 \%$ and $90 \%$ and with a side length of $1 \mathrm{~mm}$ are obtained by varying the diameter of the pores. A sample of $50 \%$ porosity is shown in Figure 2a. A resolution of $400^{3}$ voxels is used for constructing the $3 \mathrm{D}$ binary image of the microstructure together with a maximum Delaunay radius of $1 / 50 \mathrm{~mm}$ for the meshing procedure. The thermal conductivity of the matrix and the air-filled pores are set to respectively $1 \mathrm{~W} / \mathrm{mK}$ and 0.025 $\mathrm{W} / \mathrm{mK}$, the temperature difference across the sample to $10 \mathrm{~K}$.

The resulting effective thermal conductivities of the samples are calculated using Eq. 1, and are shown in Figure 3 as a function of porosity. They are compared with solutions of an analytical approximation derived by McKenzie et al. (1978) for the effective conductivity of fcc sphere packings up to their maximum porosity of $74 \%$. The analytical approximation still neglects however higher order terms, resulting in deviations from the correct solution at high porosities. Therefore, this analytical reference solution is complemented with numerical simulations performed directly in COMSOL. A representative unit cell of the fcc structure shown in Figure $2 b$ is modelled, using the program's own geometry and mesh creation functions. These results are also shown in Figure 3, together with the relative error $\eta$ between the model and this numerical reference solution.

The pore-scale model agrees very well with both of the reference solutions until a porosity of about $60 \%$, showing relative errors of less than $2 \%$. As expected, above $60 \%$ porosity the analytical approximation gets

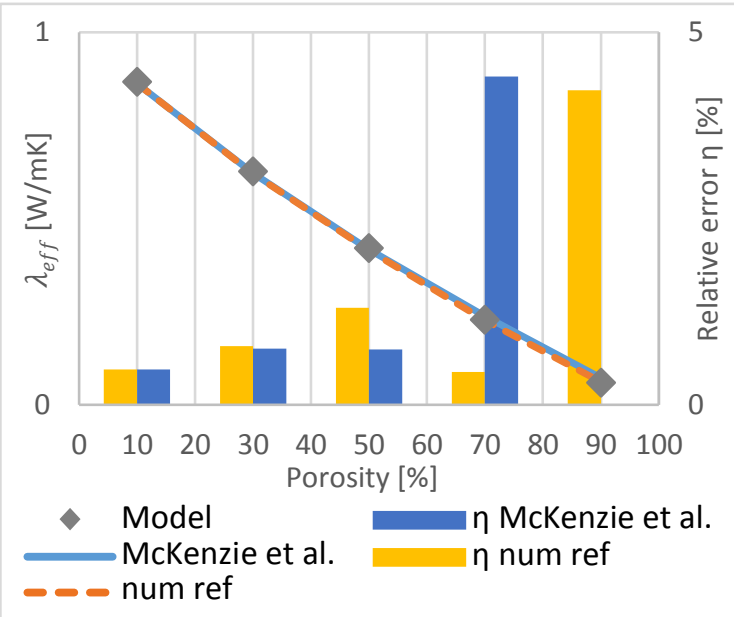

Figure 3: Verification of model with reference solutions.

less accurate showing larger discrepancies with the numerical reference model. The pore-scale model still performs very good though when compared with the numerical reference model, resulting in relative errors of less than $5 \%$ for porosities up to $90 \%$.

\section{Influence of resolution and mesh size}

The accuracy of the simulations depends strongly on a correct approximation of the porous structure by the $3 \mathrm{D}$ image and the extracted finite element mesh. This is affected by the model through the resolution and maximum mesh element size. To investigate the influence of both parameters, 9 unit cells with different porosities of the face centred cubic pore structure are simulated using the voxel-image-based model. Each sample is modelled using 3 different resolutions $\left(25^{3}, 50^{3}\right.$ and $\left.100^{3}\right)$ and 3 different maximum mesh sizes $\left(L_{\text {sample }} / 10, L_{\text {sample }} / 25\right.$ and $\left.L_{\text {sample }} / 50\right)$. The results are compared with the numerical reference model of the unit cell modelled in COMSOL. The relative errors are shown in Figure 4 as a function of porosity, resolution and mesh size.

As expected, a finer resolution and smaller mesh size generally lead to more accurate results. Resolution

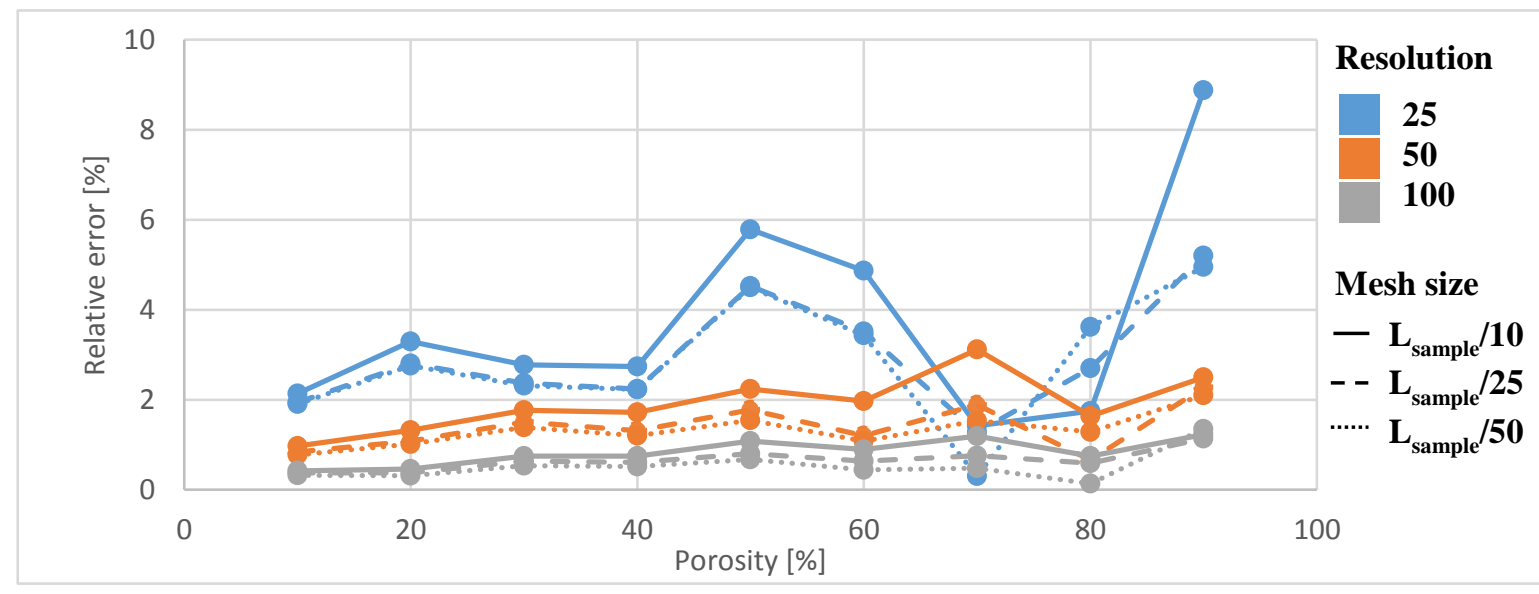

Figure 4: Influence of porosity, resolution and mesh size on accuracy. 
Postprint: Van De Walle W, Janssen H. 2016. Thermal conductivity prediction model for porous building blocks,

seems to be the most important parameter, with a resolution of $50^{3}$ or higher leading to relative errors lower than $5 \%$ for every studied porosity and mesh size. Furthermore, it appears that high porosity samples require a finer resolution. This phenomenon could be attributed to the matrix walls becoming thinner at higher porosities, which means they get represented by a relatively smaller amount of voxels leading to a poor conformity between the mesh and the geometry. These findings should be considered when applying the model in future studies.

\section{INCORPORATION OF RADIATIVE HEAT TRANSFER}

Radiative heat transfer between the pore walls can play an important role in the total heat flow through the material, particularly in materials with high porosity, at elevated temperatures, or with a low thermal conductivity gas in the pores (i.e. vacuum insulation). However, due to it's modelling complexity and $4^{\text {th }}$ order terms, it is often neglected to simplify simulations, leading to underestimations of the real ETC as pointed out by Wang \& Pan (2008). Simple corrections for the neglect of thermal radiation are then often made by adding a macroscale effective radiative conductivity to the effective thermal conductivity:

$$
\lambda_{\text {eff }}=\lambda_{\text {cond,macro }}+\lambda_{\text {rad,macro }}
$$

The value for $\lambda_{\text {rad,macro }}$ is usually obtained via either a formula based on averaged microscopic properties or via an experimental test defining a mean extinction coefficient. However, both methods are often not very accurate and the experimental parameters are cumbersome to obtain. Furthermore, the mutual influence of conductive and radiative heat transfer at the microscale is not taken into account.

To overcome the aforementioned limitations, the model developed in this study includes radiation directly at the pore scale. Classic thermal radiation modelling methods using view factors or Monte Carlo beams would however require unreasonable amounts of both CPU time and RAM memory due to the large total pore surface area. Therefore, a method introduced by Loeb (1954) is adopted and expanded. Based on the analogy with radiative heat transfer between parallel plates, he described the radiative heat transfer in a pore as a conductive process by defining an equivalent radiative thermal conductivity $\lambda_{\text {rad,pore }}$ at the pore scale as a function of the pores characteristics:

$$
\lambda_{\text {rad,pore }}=4 * \epsilon * \sigma * d_{\max } * \gamma * T^{3}
$$

$\epsilon=$ the radiative emissivity of the matrix walls; $\sigma=$ the stefan-boltzmann constant; $\mathrm{T}=$ the mean temperature of the pore in Kelvin; $\mathrm{d}_{\max }=$ the maximum distance inside the pore; $\gamma=$ a geometrical factor.

Loeb analytically determined the geometrical factor $\gamma$ to be $2 / 3$ for spherical pores and $\pi / 4$ for cylindrical pores perpendicular to the heat flow. This $\lambda_{\text {rad,pore }}$ can subsequently be added to the thermal conductivity of the gas inside the respective pore (i. e. $0.025 \mathrm{~W} / \mathrm{mK}$ for air). Hence, radiation is incorporated locally at the microscale, while maintaining a feasible simulation model. This method was further investigated by Bakker et al. (1995), numerically determining the geometrical factor for a range of oblate ellipsoid shaped pores. His results showed values for $\gamma$ varying from 0.45 to 0.66 , hereby demonstrating the large dependence of the geometrical factor on the pore geometry. However, until now a clear relation between the pore's geometrical parameters and the geometrical factor $\gamma$ is still lacking, hence impeding the correct incorporation of radiative heat transfer at the microscale.

Therefore, this paper extends the studies of Loeb and Bakker with a large range of elliptic and ellipsoidal pores, considering the fact that most pores inside porous building materials can effectively be approximated using ellipsoids. An analytical formula relating the geometrical factor to the pore's geometry is proposed.

\section{$2 D$ calculation of radiation in pores}

As a stepping stone for more complex 3D simulations, we start with 2D simulations of radiative heat transfer in elliptic pores. The equivalent geometrical factor $\gamma$ is calculated performing a set of simulations on a square containing just one such elliptic pore. Different ellipses are studied by varying the 3 different geometrical parameters (the long diameter ' $a$ ', the ratio of the diameters ' $b / a$ ' and the angle ' $\alpha$ ' with the horizontal) shown in Figure 5.

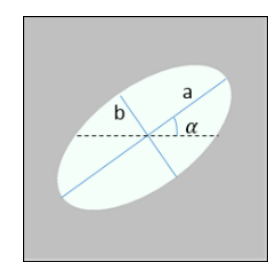

Figure 5: View on the elliptic pore parameters

The opposing sides of the squares are assigned with a temperature diffence of $1 \mathrm{~K}$, while the matrix and air thermal conductivity are set to $1 \mathrm{~W} / \mathrm{mK}$ and 0.025 $\mathrm{W} / \mathrm{mK}$. Simulations are performed at 7 different mean temperatures. In this first study, only an emissivity of 0.9 is considered, although Fitzgerald \& Strieder (1997) have shown that the pore scale radiative conductivity does not behave completely linear with emissivity for all pore shapes. This will be adressed in future studies, together with the influence of the matrix's thermal conductivity. Caution should therefore be applied when extending the results to low emissivities. The parameters and studied values are listed in Table 1. All mutual combinations are studied, hence covering a very broad range of possible elliptic pore shapes. 
Postprint: Van De Walle W, Janssen H. 2016. Thermal conductivity prediction model for porous building blocks, Bauphysik, 38(6):340-347.

Table 1:

Studied parameters for 2D elliptic pores.

\begin{tabular}{|l|l|}
\hline \multicolumn{1}{|c|}{ PARAM. } & \multicolumn{1}{c|}{ VALUES } \\
\hline $\mathrm{a}[\mathrm{mm}]$ & $0.1-0.25-0.5-0.75-1-1.5-2$ \\
\hline $\mathrm{b} / \mathrm{a}[-]$ & $0.33-0.5-0.75-1$ \\
\hline$\alpha\left[^{\circ}\right]$ & $0-18-36-54-72-90$ \\
\hline$T_{\text {mean }}[\mathrm{K}]$ & $263-273-283-293-303-313-323$ \\
\hline
\end{tabular}

The heat flow through the square is simulated including radiative heat transfer inside the elliptical pore using the view factor method in COMSOL. The effective thermal conductivity $\lambda_{\text {eff,with direct rad }}$ of the square is obtained via the Fourier formula. Subsequently, the same simulation is performed without including radiative heat transfer, resulting in a $\lambda_{\text {eff,no rad }}$. Finally, a search algorithm determines the equivalent $\lambda_{\text {rad,pore,numerical }}$ that should be added to the thermal conductivity of the air inside the pore so the relative error defined in Eq. 4 is smaller than $0.1 \%$ :

$$
\frac{\lambda_{\text {eff,with direct rad }}-\lambda_{\text {eff,with conductive rad }}}{\lambda_{\text {eff,with direct rad }}-\lambda_{\text {eff,no rad }}}
$$

The determined values for $\lambda_{\text {rad,pore,numerical }}$ show to be perfectly related to the temperature according to the $\mathrm{T}^{3}$ term in Loeb's formula. For every pore the resulting geometrical factor $\gamma$ can then be calculated as:

$$
\gamma_{\text {pore }}=\frac{\lambda_{\text {rad,pore, } \text { numerical }}}{4 * \epsilon * \sigma * T^{3} * d_{\max }}
$$

As expected, it was found that $\gamma$ depends strongly on the geometrical parameters ' $\mathrm{a}$ ', 'b/a' and ' $\alpha$ '. To avoid calculating these 3 parameters for every pore in future studies, a new, more easy to calculate factor is introduced combining the effect of all 3 geometrical parameters:

$$
S_{f, i}=\frac{M H_{\text {pore }}}{M D_{\text {pore }}}
$$

$S_{f, i}=$ the slenderness factor in direction ' $i$ ' of the cube $[-] ; \mathrm{i}=$ the direction of the heat flow; $M H_{\text {pore }}=$ the mean heigth of the pore, calculated perpendicular to the direction ' $\mathrm{i}$ ' $[\mathrm{m}] ; M D_{\text {pore }}=$ the mean distance of the pore, calculated parallel to the direction ' $\mathrm{i}$ ' $[\mathrm{m}]$.

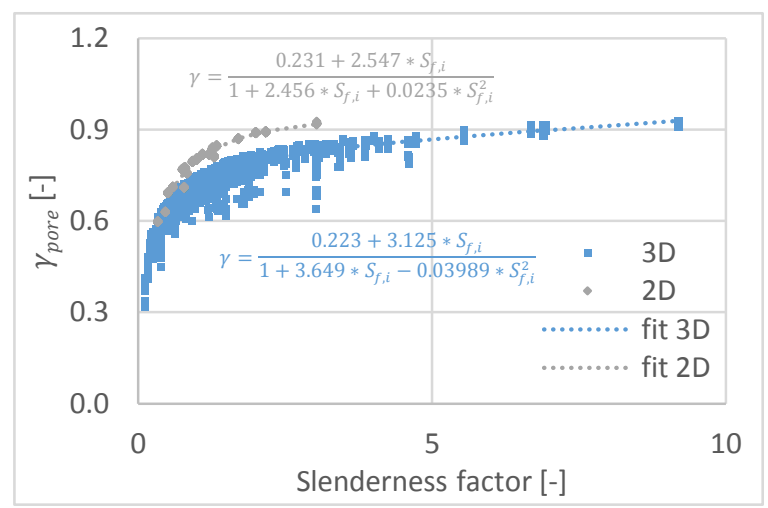

Figure 6: Relation between $\gamma$ pore and the slenderness factor (for $2 D$ and for $3 D$ pores)
A low slenderness value means that the pore is elongated in the direction of the heat flow while a high value means the pore is elongated in a direction perpendicular to the heat flow. A circular pore has by definition a slenderness of 1 . Important to notice is that the slenderness of a pore depends on the direction of the heat flow. Furthermore, we propose to replace the maximum distance ' $d_{\max }$ ' used in Loeb's formula (Eq. 3) with the mean distance $M D_{\text {pore }}$, for more consistency in the formula. This is also adapted in the calculation of $\gamma$ for every pore. The relation between $\gamma$ and the slenderness factor is shown in Figure 6.

It can be seen that a very close relation exists between the factor $\gamma_{\text {pore }}$ and the slenderness factor. The results furthermore confirm the intuitive idea that a vertically elongated ellips, with a high slenderness factor, has a larger $\gamma$ and hence higher radiative heat transfer. The fitted relation between both is used to adapt Loeb's formula to:

$$
\begin{gathered}
\lambda_{\text {rad,pore,analytical }}= \\
4 * \epsilon * \sigma * T^{3} * M D_{\text {pore }} * \\
\frac{0.231+2.547 * S_{f, i}}{1+2.456 * S_{f, i}+0.0235 * S_{f, i}^{2}}
\end{gathered}
$$

Figure 7 compares for all the pores the analytical and numerical calculation of $\lambda_{\text {rad,pore }}$, showing good agreement.

\section{D calculation of radiation in pores}

The same methodology as described for $2 \mathrm{D}$ pores is applied for the 3D case. A cube containing one ellipsoidal pore is simulated modelling radiative heat transfer directly using view factors and subsequently determining the equivalent radiative thermal conductivity of the pore. The parameters in 2D are extended with the length $\mathrm{c}$ of the short axis in the $3^{\text {rd }}$ dimension, and 2 other angles to allow the pore to have any form and orientation. Only one temperature of 293 $\mathrm{K}$ is studied since the $2 \mathrm{D}$ calculations confirmed already the adoption of $\mathrm{T}^{3}$ in Loeb's formula. For the emissivity, again only a value of 0.9 is used, keeping in mind the remarks made under the $2 \mathrm{D}$ calculations.

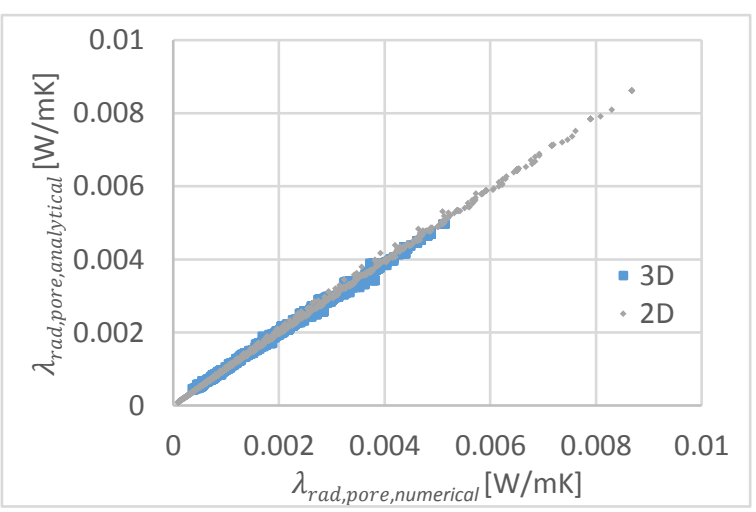

Figure 7: Comparison of numerical and analytical calculation of $\lambda$ rad,pore (for $2 D$ and $3 D$ pores) 
Postprint: Van De Walle W, Janssen H. 2016. Thermal conductivity prediction model for porous building blocks, Bauphysik, 38(6):340-347.

The combination of all the parameters and their studied values are listed in Table 2 .

Table 2:

Studied parameters for $3 D$ ellipsoidal pores.

\begin{tabular}{|l|l|}
\hline \multicolumn{1}{|c|}{ PARAM. } & \multicolumn{1}{c|}{ VALUES } \\
\hline $\mathrm{a}[\mathrm{mm}]$ & $0.5-1-1.5-2$ \\
\hline $\mathrm{b} / \mathrm{a}[-]$ & $0.33-0.5-0.75-1$ \\
\hline $\mathrm{c} / \mathrm{a}[-]$ & $0.33-0.5-0.75-1$ \\
\hline$\alpha_{1}\left[^{\circ}\right]$ & $0-30-60-90$ \\
\hline$\alpha_{2}\left[^{\circ}\right]$ & $0-30-60-90$ \\
\hline$\alpha_{3}\left[^{\circ}\right]$ & $0-30-60-90$ \\
\hline
\end{tabular}

The slenderness factor is now extended to the $3^{\text {rd }}$ dimension by incorporating the mean width $M W_{\text {pore }}$ of the pore:

$$
S_{f, i}=\frac{M H_{\text {pore }} * M W_{\text {pore }}}{\left(M D_{\text {pore }}\right)^{2}}
$$

The geometrical factor $\gamma$ can again be calculated using Eq. 5 where again the distance of the pore is replaced with the mean distance. A good relation between the slenderness factor and $\gamma$ appears also for 3D pores, as shown in Figure 6.

The Loeb formula can be expanded in an analogous manner as the $2 \mathrm{D}$ case:

$$
\begin{gathered}
\lambda_{\text {rad,pore,analytical }}= \\
4 * \epsilon * \sigma * T^{3} * M D_{\text {pore }} * \\
\frac{0.223+3.125 * S_{f, i}}{1+3.649 * S_{f, i}-0.03989 * S_{f, i}^{2}}
\end{gathered}
$$

Figure 7 compares for all the pores the analytical and numerical calculation of $\lambda_{\text {rad,pore }}$. Good agreement is again obtained demonstrating the validity of the analytical approximation.

\section{Implementation in Matlab}

The adapted Loeb formula is used to assign an equivalent radiative thermal conductivity to every pore in the sample based on the pore's slenderness factor. A number of Matlab scripts have been implemented with the purpose of calculating this slenderness factor for every pore.

The first step consists of splitting the pore space into separated pore clusters. Indeed, in high porosity materials often a large part of the pores are interconnected with smaller connection zones. However, when the opening width of the connection between two pore clusters is relatively small, thermal radiation travelling from one cluster to the other can effectively be neglected. Hence the 2 pore clusters should be separated and a different slenderness factor for both clusters should be calculated. The splitting of the pores is executed according to the watershed-based procedure described in Morpho+ (Brabant et al. 2011), by calculating the distance transform of the binary image matrix. This procedure will split all the pores at their narrowest point, resulting in a set of completely disconnected pores. However, when pore clusters were originally connected via a relatively large opening width, their splitting is not desirable since thermal radiation travelling between 2 pore clusters will have an important impact in this case. Therefore, the rejoining procedure described in Morpho+ is also implemented. This procedure first calculates the radii of the maximum inscribed balls of 2 originally connected pores. Subsequently, the largest of both radii is compared to the radius of the maximum inscribed circle in the connection zone between both pores. If the ratio of these radii is larger than a set value (the rejoin factor $R_{f}$ ), the pores are rejoined:

$$
\frac{r_{\text {inscribed circle of connection }}}{\max \left(r_{\text {inscribed balls of pores }}\right)}>R_{f}
$$

If the rejoin factor is set to 0 , all separated pores will be rejoined, if set to 1 all pores will stay separated. The binary image matrix is hence transformed to an image matrix consisting of several pore clusters, each arising from a number of pores that pass the rejoining test. For every pore cluster the slenderness factor is calculated using Eq. 8, which is finally used to calculate the equivalent radiative thermal conductivity in every pore cluster. Since these conductivities can simply be added to the thermal conductivity of the gas in the pores, the whole simulation procedure remains the same as described in the first section.

\section{PRELIMINARY INVESTIGATIONS USING THE MODEL}

The model is used to investigate the impact of both the difference between 2D and 3D simulations and the influence of thermal radiation on the total heat transfer. A synthetic sample is generated, having a porosity of $81.73 \%$, a side length of $1 \mathrm{~cm}$ and a pore size distribution as shown in Figure 8 (left). The resolution is set to $600^{3}$, the mesh size parameter to $\mathrm{L}_{\text {sample }} / 50$. The sample is shown in Figure 8 (right).
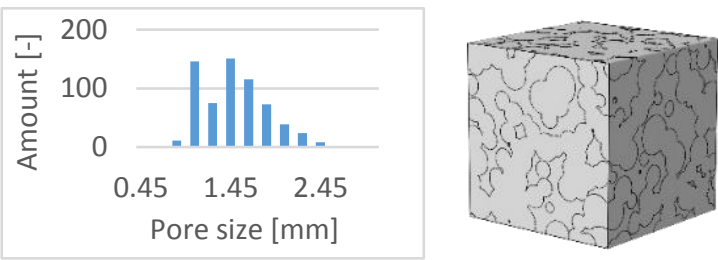

Figure 8: The pore size distribution (left) and a render of the investigated sample (right)

The effective thermal conductivity excluding thermal radiation is calculated with the model, resulting in a value of $0.064 \mathrm{~W} / \mathrm{mK}$.

\section{D versus 3D calculations}

For simplification, a lot of calculation models use a 2D approach with an image acquired via generating 
Postprint: Van De Walle W, Janssen H. 2016. Thermal conductivity prediction model for porous building blocks,

algorithms or scanning electron microscopy as input. This is however a strong simplification of the real microstructure and the pathways of the heat flow through the material. This is demonstrated on the generated 3D sample: five equidistant 2D slices are cut through the sample, starting and finishing with the bottom and upper slice. The middle slice is shown in Figure 9.
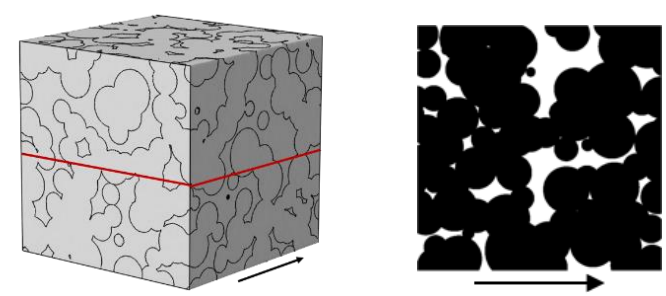

Figure 9: Location of the slice (left) and 2D view of the slice (right)

The effective thermal conductivity of every slice is calculated using the model, excluding thermal radiation. The resulting ETC of the respective slices are shown in Figure 10.

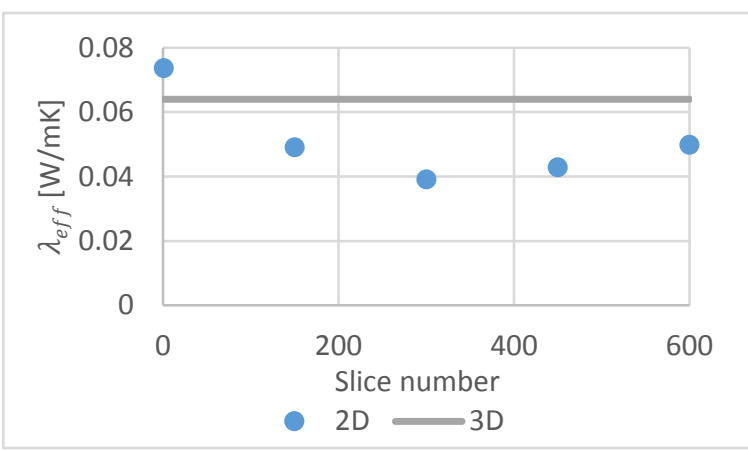

Figure 10: $2 D$ versus $3 D$ calculation.

A first conclusion is that the $2 \mathrm{D}$ calculated values differ a lot from each other, due to the different porosity and microstructure of each slice. Furthermore, most of the values are lower than the $3 \mathrm{D}$ value, and so is also the average of the $2 \mathrm{D}$ values. This is due to the fact that in $3 \mathrm{D}$ the heat flow can pass the $2 \mathrm{D}$ obstructions in the $3^{\text {rd }}$ dimension. Hence, 2D calculations will result in a strong underestimation of the true effective thermal conductivity.

\section{Influence of radiation}

The effect of radiative heat transfer on the ETC of the synthetic sample of Figure 8 is investigated. This is done through the method described before, incorporating radiation at the pore level. A rejoin factor of 0.5 is applied, leading to a subdivision of the pores in 13 pore clusters. For each cluster, the geometrical factor $\gamma$ is calculated using the cluster's slenderness factor previously defined.

To reduce computation time, the mean sample temperature is used in the adapted Loeb formula instead of the local temperature. With the temperature difference across the sample being set to $10 \mathrm{~K}$, this should induce only a very small error. The simulation is performed at 3 different mean temperatures: $273 \mathrm{~K}$, $293 \mathrm{~K}$ and $313 \mathrm{~K}$. The results are summarized in Table 3 , together with the ETC of the sample excluding thermal radiation. The relative difference with this value is also shown.

Table 3:

ETC at several temperatures, with and without radiation

\begin{tabular}{|l|l|l|}
\hline TMEAN [K] $_{\text {MTC }}$ & EW/MK] & REL. DIFFERENCE \\
\hline No radiation & 0.064 & N.A. \\
\hline 273 & 0.0713 & $10.24 \%$ \\
\hline 293 & 0.0730 & $12.33 \%$ \\
\hline 313 & 0.0749 & $14.55 \%$ \\
\hline
\end{tabular}

It is clear that thermal radiation has a non-negligible influence. Even at a mean temperature of $273 \mathrm{~K}$, the ETC is found to be $10 \%$ higher than the one neglecting thermal radiation. As expected, this increases with increasing temperature. It is furthermore expected that the influence will be even larger at higher porosities.

The model results are compared with two analytical macroscale calculations of the thermal radiation. The first is based on the calculation of the mean extinction coefficient following an empirical formula from $\mathrm{Hsu}$ \& Howell (Howell 2000) for open-celled reticulated ceramic foams:

$$
\begin{aligned}
& \lambda_{\text {rad,macro }}=\frac{16 * \sigma * T^{3}}{3 * \kappa} \\
& \kappa=\frac{3}{d_{\text {pore,mean }}} *(1-\phi)
\end{aligned}
$$

The other method is a simplified calculation for closed cell materials derived by Batty et al. (1984):

$$
\lambda_{\text {rad,macro }}=\frac{4 * \sigma * T^{3} * d_{\text {pore }, \text { mean }}}{\frac{2}{\epsilon}-1}
$$

The values for $\lambda_{\text {rad,macro }}$ are summed with the thermal conductivity of the sample calculated without thermal radiation $(0.064 \mathrm{~W} / \mathrm{mK})$. The results of the two analytical macroscale approximations are shown in Figure 11 as a function of mean temperature, alongside the results obtained with the model.

As expected, the model results lie in between both approximations, since the synthetic sample is neither completely closed- or open-celled. The much higher values for the Hsu \& Howell model can be attributed to the fact that their formula is derived for very open porous ceramic foams. Though further verification studies are still needed, this is already a strong first indication of the possibilities of this microscale approach. 
Postprint: Van De Walle W, Janssen H. 2016. Thermal conductivity prediction model for porous building blocks,

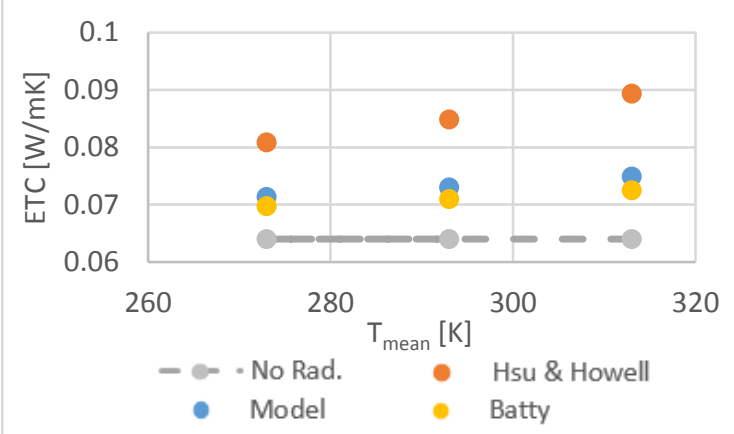

Figure 11: ETC at several temperatures, with and without radiation

\section{CONCLUSION}

This paper introduced a model framework for performing 3D heat simulations on microstructures of porous samples. Good agreement was found for an elementary type of pore structure for porosities between $10 \%$ and $90 \%$. The method of Loeb for incorporating thermal radiation at the microscale has been implemented and extended on the basis of a broad set of pore scale radiative heat transfer simulations. A slenderness factor has been introduced, allowing for an accurate calculation of Loeb's geometrical factor. This makes it possible for including radiative heat transfer at the pore scale, based on local structural characteristics instead of Loeb's non-intuitive factor $\gamma$. The model is subsequently used to show the discrepancy between 2D and 3D simulations, and to show the importance of including thermal radiation.

\section{ACKNOWLEDGEMENT}

Research funded by a Ph. D. grant of the Agency for Innovation by Science and Technology (IWT).

\section{REFERENCES}

Alliez, P. et al., 2015. 3D Mesh Generation. In CGAL User and Reference Manual. CGAL Editorial Board.

Bakker, K., Kwast, H. \& Cordfunke, E.H.P., 1995. The contribution of thermal radiation to the thermal conductivity of porous UO2. Journal of Nuclear Materials, 223, pp.135-142.

Batty, W.J., Probert, S.D. \& O’Callaghan, P.W., 1984. Apparent thermal conductivities of highporosity cellular insulants. Applied Energy, 18(2), pp.117-135.

Brabant, L. et al., 2011. Three-dimensional analysis of high-resolution X-ray computed tomography data with Morpho+. Microscopy and Microanalysis, 17(2), pp.252- 263.

Carson, J.. et al., 2003. An analysis of the influence of material structure on the effective thermal conductivity of theoretical porous materials using finite element simulations. International Journal of Refrigeration, 26(8), pp.873-880.

Chen, J. et al., 2015. Evaluation of thermal conductivity of asphalt concrete with heterogeneous microstructure. Applied Thermal Engineering, 84, pp.368-374.

Clyne, T.W. et al., 2006. Porous materials for thermal management under extreme conditions. Philosophical transactions. Series A, Mathematical, physical, and engineering sciences, 364(1838), pp.125-146.

Fang, Q. \& Boas, D.A., 2009. Tetrahedral mesh generation from volumetric binary and gray scale images. In Proceedings IEEE International Symposium on Biomedical Imaging. pp. 1142-1145.

Fitzgerald, S. \& Strieder, W., 1997. Radiation heat transfer across a compressed spheroidal cavity. AIChE Journal, 43(9), pp.2368-2372.

Gaiselmann, G. et al., 2014. Quantitative relationships between microstructure and effective transport properties based on virtual materials testing. AIChE Journal, 60(6), pp.1983-1999.

Howell, J.R., 2000. Part VI: Radiative transfer in porous media. In K. Vafai, ed. Handbook of Porous Media. pp. 663-698.

Loeb, A., 1954. Thermal Conductivity: VIII, A theory of thermal conductivity of porous materials. Journal of the American Ceramic Society, 37(2), pp.96-99.

McKenzie, D.R., McPhedran, R.C. \& Derrick, G.H., 1978. The conductivity of lattices of spheres II. The body centred and face centred cubic lattices. Proceeding of the Royal Society of London. Series A, Mathematical and Pysical Sciences, 362(1709), pp.211-232.

Randrianalisoa, J. \& Baillis, D., 2014. Thermal conductive and radiative properties of solid foams: Traditional and recent advanced modelling approaches. Comptes Rendus Physique, 15(8-9), pp.683-695.

She, W., Zhang, Y. \& Jones, M.R., 2014. Threedimensional numerical modeling and simulation of the thermal properties of foamed concrete. Construction and Building Materials, 50, pp.421-431.

Wang, M. \& Pan, N., 2008. Modeling and prediction of the effective thermal conductivity of random open-cell porous foams. International Journal of Heat and Mass Transfer, 51(5-6), pp.13251331. 